Supplement of Biogeosciences Discuss., 12, 17125-17152, 2015

http://www.biogeosciences-discuss.net/12/17125/2015/

doi:10.5194/bgd-12-17125-2015-supplement

(C) Author(s) 2015. CC Attribution 3.0 License.

(c) (1)

Supplement of

\title{
Photochemical mineralisation in a humic boreal lake: temporal variability and contribution to carbon dioxide production
}

M. M. Groeneveld et al.

Correspondence to: B. Koehler (birgit.koehler@ebc.uu.se)

The copyright of individual parts of the supplement might differ from the CC-BY 3.0 licence. 


\section{Supplementary material}
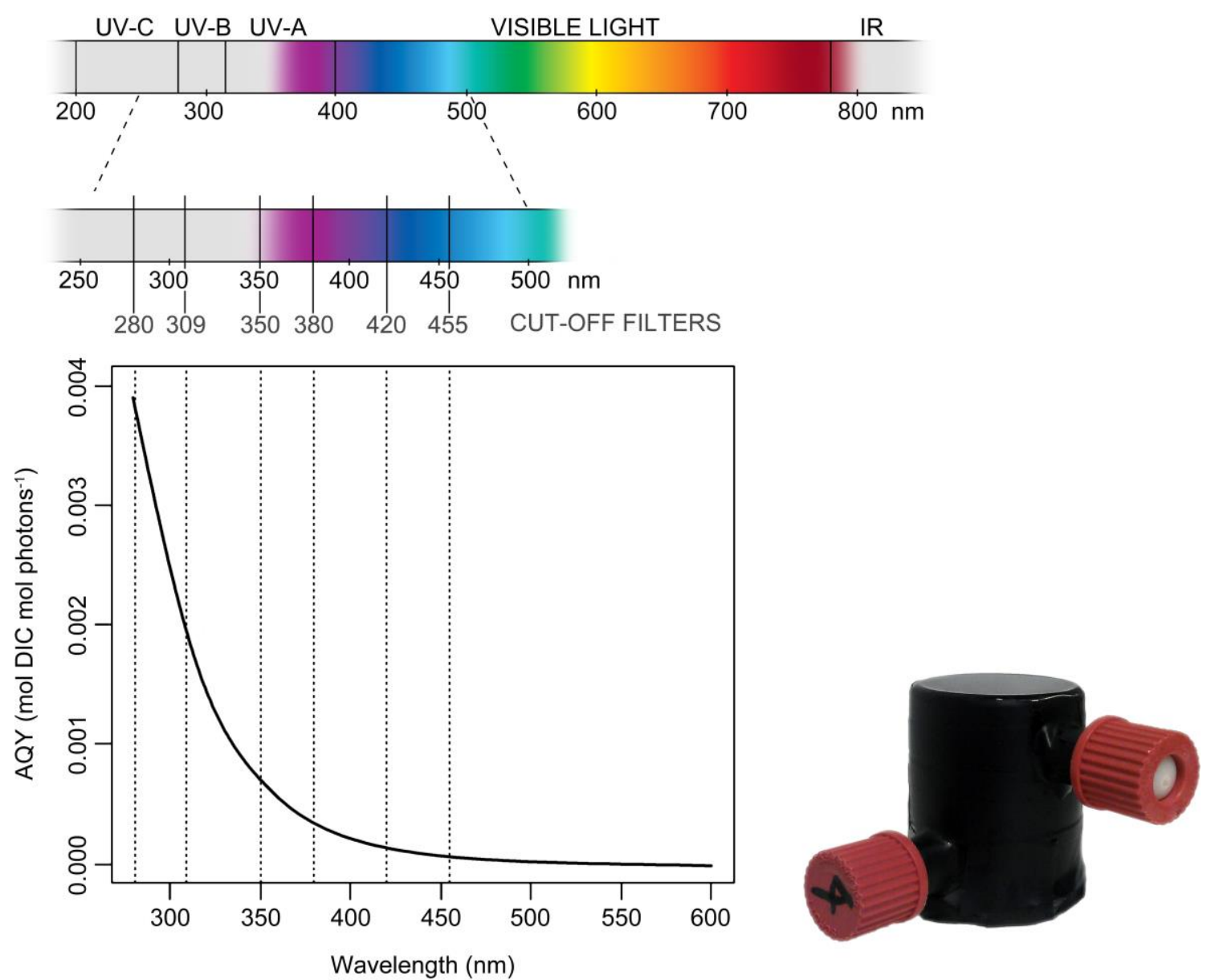

Figure S1. Determination of the apparent quantum yield (AQY) spectrum. a: Glass incubation vial with quartz top, sides covered with black insulation tape to avoid irradiance to enter laterally. b: Position of the cut-off filters with respect to the irradiance spectrum, and an exemplary AQY spectrum calculated based on the photochemical DIC production and CDOM-absorbed photons for each cut-off filter. 

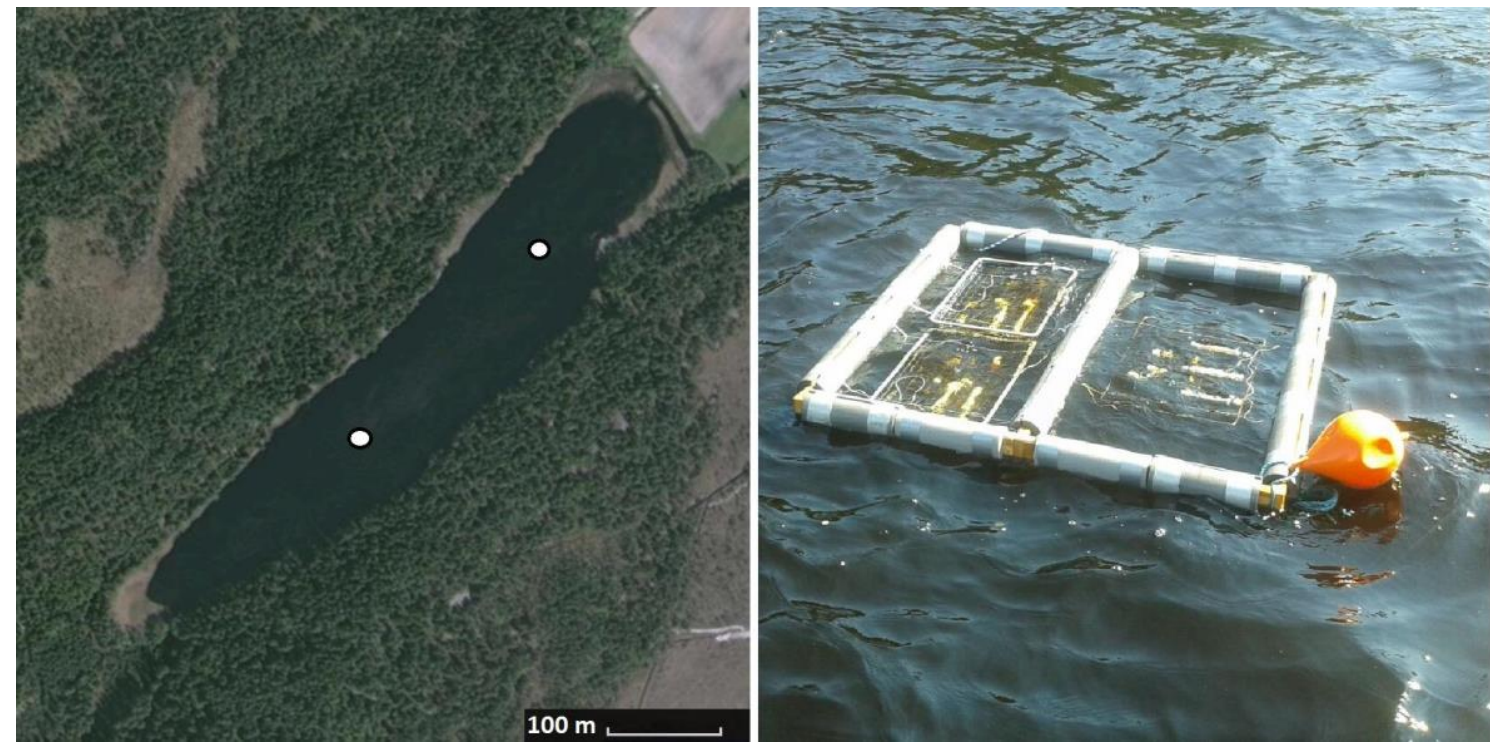

Figure S2. a: Aerial photo of lake Erssjön, indicating the locations of the two frames used during in-situ measurement of DIC photoproduction (image obtained from Google maps; Imagery (C2015 Lantmäteriet/Metria, Map data (C2015 Google). $\mathrm{b}$ : Floating frame with the quartz and control tubes positioned at three different water depths. 


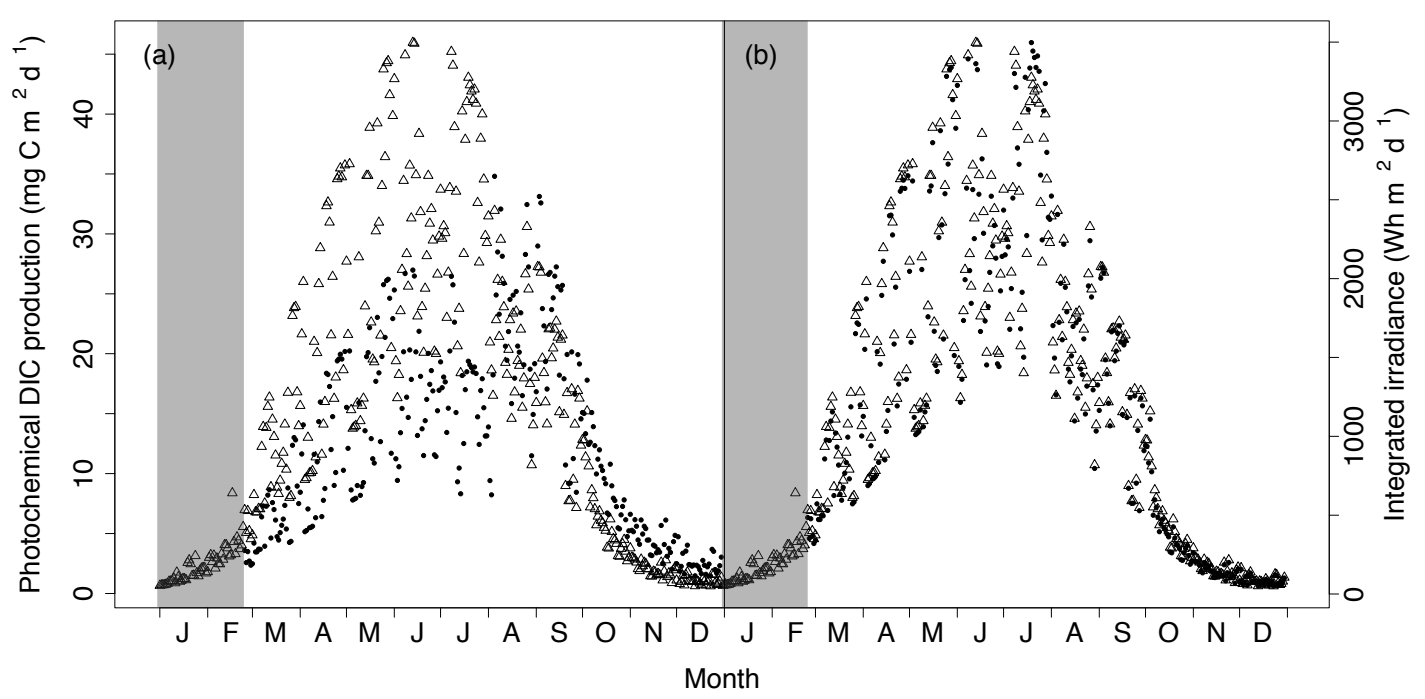

Figure S3. Daily photochemical DIC production rate (dots) and downwelling scalar irradiance just below the water surface (triangles; integrated over the wavelength range 280-600 nm) for 2014. The graphs show results from two model parameterisations using (a) the monthly measured AQY spectra for month-long time periods around the sampling date or (b) the pooled AQY spectrum across the six monthly measurements during the open water season. 